\title{
Simulated climate change provokes rapid genetic change in the Mediterranean shrub Fumana thymifolia
}

\author{
ALISTAIR S. JUMP*, JOSEP PEÑUELAS*, LAURA RICO*†, ELISENDA RAMALLO*, \\ MARC ESTIARTE*, JOSÉ A. MARTÍNEZ-IZQUIERDO† and FRANCISCOLLORET \\ *Unitat d'Ecofisiologia i Canvi Global CSIC-CEAB-CREAF, CREAF (Centre de Recerca Ecològica i Aplicacions Forestals), Edifici C, \\ Universitat Autònoma de Barcelona, 08193 Bellaterra (Barcelona), Catalonia, Spain, †Departament de Genètica Molecular, Consorci \\ Laboratori CSIC-IRTA de Genetica Molecular Vegetal, c/Jordi Girona 18-26, 08034 Barcelona, Catalonia, Spain, †Unitat d’Ecología, \\ Dept. Biologia Animal, Biologia Vegetal i Ecologia, i CREAF (Centre de Recerca Ecologica i Aplicacions Forestals), Edifici C, \\ Universitat Autònoma de Barcelona, 08193 Bellaterra (Barcelona), Catalonia, Spain
}

\begin{abstract}
Rapid climate change will impose strong directional selection pressures on natural plant populations. Climate-linked genetic variation in natural populations indicates that an evolutionary response is possible. We investigated such a response by comparing individuals subjected to elevated drought and warming treatments with individuals establishing in an unmanipulated climate within the same population. We report that reduction in seedling establishment in response to climate manipulations is nonrandom and results from the selection pressure imposed by artificially warmed and droughted conditions. When compared against control samples, high single-locus genetic divergence occurred in drought and warming treatment samples, with genetic differentiation up to 37 times higher than background (mean neutral locus) genetic differentiation. These loci violate assumptions of selective neutrality, indicating the signature of natural selection by drought. Our results demonstrate that rapid evolution in response to climate change may be widespread in natural populations, based on genetic variation already present within the population.
\end{abstract}

Keywords: adaptation, AFLP, demography, drought, environmental change, genome scan, population genomics, warming

Received 1 June 2007; revised version received 1 October 2007 and accepted 5 October 2007

\section{Introduction}

Predictions of the effects of climatic change at species level have often been based on monitoring changes in local abundance (Lewis-Smith, 1994; Alward et al., 1999) or on the geographic range of particular taxa (Parmesan et al., 1999; Walther et al., 2001; Warren et al., 2001; Peñuelas \& Boada, 2003). However, in addition to forcing changes in species' distributions and their presence and abundance within communities, current climatic changes are expected to impose strong directional selection pressures on natural populations (Davis \& Shaw, 2001; Davis et al., 2005; Jump \& Peñuelas, 2005;

\footnotetext{
Correspondence: Present address: Alistair S. Jump, Environment Department, University of York, Heslington, York YO10 5DD, UK, tel. +44 1904 434780, fax +44 1904 432998,

e-mail: aj523@york.ac.uk

(C) 2008 The Authors

Journal compilation (C) 2008 Blackwell Publishing Ltd
}

Thomas, 2005; Reusch \& Wood, 2007; Strauss et al., in press).

Accumulating evidence now demonstrates that evolutionary responses to the selection pressures imposed by climate change can and do occur. Climate-change driven shifts have been reported in clinal patterns of isozyme polymorphisms in Drosophila (Umina et al., 2005) and photoperiodic response in the pitcher-plant mosquito, Wyeomyia smithii (Bradshaw \& Holzapfel, 2001), while earlier springs have led to selection for earlier breeding in the red squirrel Tamiasciurus hudsonicus in western Canada (Réale et al., 2003). Episodes of drought may select for increased body size in Darwin's finches (Boag \& Grant, 1981; Grant \& Grant, 2002), while in the annual plant, Brassica rapa, a recent multiyear drought episode has led to rapid evolution in this species toward an earlier flowering and, therefore, drought-avoiding phenotype (Franks et al., 2007). Ge- 
netic variability associated with particular environmental conditions has been reported for plant species as diverse as cereal crops and forest trees (reviewed by Jump \& Peñuelas, 2005). Given the existence of such climate-related genetic variability within natural populations, adaptation to changes in climate may be widespread, and could complicate prediction of species' range changes in response to climate change if migration is also accompanied by rapid evolutionary change (Jump \& Peñuelas, 2005).

Using a genome scan, a population genomic analysis that compares genetic divergence between populations at individual molecular marker loci with a genomewide average, the signature of selection can be detected (Luikart et al., 2003; Beaumont, 2005; Storz, 2005). This approach has been used recently to identify evolutionary responses to disease control agents (Wooton et al., 2002) and during domestication of ancestral crop varieties (Vigouroux et al., 2002), adaptation along environmental gradients (Wilding et al., 2001; Campbell \& Bernatchez, 2004; Bonin et al., 2006; Jump et al., 2006a), and sympatric speciation in plants (Savolainen et al., 2006). Genome scans, therefore, provide an appropriate tool for determining whether an evolutionary response has occurred in populations subjected to climatic changes, if individuals can be compared from contrasting climates imposed within the same population. Long-term climate manipulation experiments, which have so far provided significant insight into likely species and community level effects of climate change, provide a unique starting point for such investigation.

Early developmental stages of plants are expected to be more sensitive to environmental constraints than adult stages, as supported by the typical pattern of decreasing risk of death from birth in plant populations (Watkinson, 1997). Assessment of seedling establishment in response to experimental climate manipulation has shown that demographic processes are sensitive to even minor climate changes, leading to changes in the presence and abundance of species within plant communities (Lloret et al., 2004). Given these demographic changes, we investigated whether reduced seedling establishment observed as a consequence of climate manipulation is a random or selective process, thereby allowing us to answer the key question: does climate change provoke evolutionary change within natural populations?

\section{Materials and methods}

\section{Site location and species description}

The experiment was performed at Garraf Natural Park, Barcelona, Spain, $\left(41^{\circ} 8^{\prime} \mathrm{N}, 1^{\circ} 54^{\prime} \mathrm{E}\right.$, at $300 \mathrm{~m}$ a.s.1.) in an early successional Mediterranean shrubland commu- nity (Peñuelas et al., 2004). The studied species, Fumana thymifolia (L.) Spach ex Webb (Cistaceae), is a small shrub that grows up to $25 \mathrm{~cm}$ high. It occurs in open shrublands around the Mediterranean Basin on calcareous soils. F. thymifolia is insect pollinated with a mixed mating system. Both outcrossed and self-pollinated flowers can bear seed, although selfing reduces seed production by more than $50 \%$ in comparison with outcrossing (F. Lloret, data not shown). Clonal reproduction was not observed. Flowering occurs in spring and summer and abundant seedling establishment occurs after autumn or winter rains. Although no detailed information on the age structure of the experimental population is available, our observations suggest that the average generation time is longer than the study period. The species F. ericoides (syn Fumana ericifolia Wallr. in Linnaea) co-occurs with F. thymifolia on the study site although these species are not known to hybridise (Castroviejo et al., 1993).

\section{Drought and warming treatments}

Drought, warming, and control treatments were applied on nine $20 \mathrm{~m}^{2}$ plots (three plot replicates by treatment). These treatments were physically intermixed within the same plant community (Beier et al., 2004; Peñuelas et al., 2004) (Fig. 1). Homogeneity of vegetation among plots in terms of aspect, slope and vegetation composition was ensured at the start of the experiment. The drought treatment consisted of automatically activated transparent plastic covers, which covered the plots in response to rainfall and retreated when rainfall stopped. The warming treatment was of

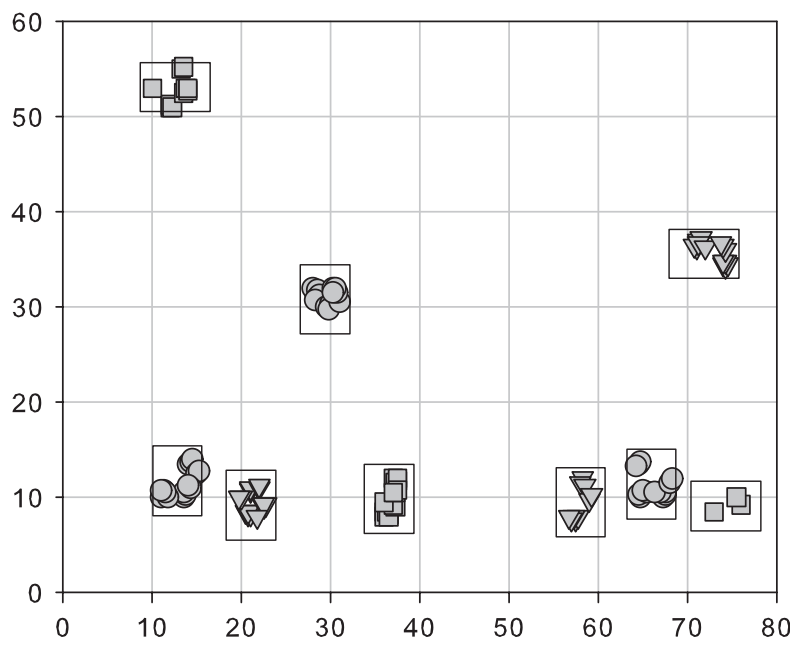

Fig. 1 Location of treatment plots and Fumana thymifolia samples. Triangles represent individuals sampled from drought treatment plots; squares, warming; circles, control. Gridlines represent $10 \mathrm{~m}$ divisions of the study site. 
similar construction, with reflective covers that reduced the loss of infra-red radiation by preventing night-time re-radiation of accumulated daytime solar energy back to the atmosphere. This form of warming treatment closely mimics that of greenhouse-gas-induced global warming (Peñuelas et al., 2004). The warming covers were automatically removed during rain events, triggered by rain sensors. Three untreated control plots included the same light scaffolding used for the warming and drought treatments, but without any covers. The warming treatment resulted in a temperature increase of ca. $1{ }^{\circ} \mathrm{C}$ and the drought treatment decreased soil moisture by ca. $20 \%$. This ongoing experiment has been active since April 1999. Details of the experimental setup have been published previously in full (Beier et al., 2004; Lloret et al., 2004; Peñuelas et al., 2004).

\section{Seedling survey and sampling}

The number of seedlings observed in the study plots was recorded annually over the experimental period following the protocol described by Lloret et al. (2004) and mean yearly $F$. thymifolia seedling density was then calculated. In February 2005, we sampled leaf tissue from all surviving seedlings that had established during the period of the experiment and experienced at least one drought season (summer) and one growth season (spring). Because no seedling establishing during the experiment had reached reproductive maturity, all sampled seedlings analysed in this study are believed to be the offspring of plants predating the climate manipulation treatments. Sampled individuals are, therefore, the first generation to experience selection during establishment as a result of the imposed climatic manipulations. All individuals sampled within the three plots of each climate treatment were pooled for subsequent population genomic analysis. The total number of individuals sampled per treatment was: drought treatment, 40 individuals; warming treatment, 29; control treatment, 45.

\section{AFLP analysis}

Approximately $0.5 \mathrm{~cm}^{2}$ leaf tissue was ground in liquid nitrogen for $30 \mathrm{~s}$ at 30 revolutions per second using a mixer mill (Tissue Lyser, Qiagen Inc., Valencia, CA, USA) and two glass beads. Genomic DNA was extracted from ground tissue using a DNeasy Plant Mini Kit (Qiagen) and quantified using a NanoDrop ND-1000 spectrophotometer running software v3.0.1 (NanoDrop Technologies, Wilmington, DE, USA) following the manufacturers instructions. AFLP analysis followed a modified version of the original protocol published by Vos et al. (1995) as detailed below.
Restriction digests and ligation of adapters. DNA $(0.5 \mu \mathrm{g})$ was digested at $37^{\circ} \mathrm{C}$ for $3 \mathrm{~h}$ with $12 \mathrm{U}$ EcoRI (Amersham, Little Chalfont, UK) and $8 \mathrm{U}$ of $\mathrm{Mse}$ (New England Biolabs, Ipswich, MA, USA) in $35 \mu \mathrm{L}$ of RL buffer (comprising $10 \mathrm{~mm}$ Tris-acetate $\mathrm{pH}$ 7.5, $10 \mathrm{~mm}$ magnesium acetate, $50 \mathrm{~mm}$ potassium acetate, and $20 \mathrm{~mm}$ DTT).

PCR adapters were ligated to the cut fragments by adding to the restriction digest $5 \mu \mathrm{L}$ of RL buffer containing $0.2 \mathrm{mM}$ ATP, $125 \mathrm{nM}$ EcoRI adapter, $1.25 \mu \mathrm{M}$ MseI adapter and 1.4U T4 DNA ligase (Roche Applied Science, Basel, Switzerland). Ligation was allowed to proceed for $3 \mathrm{~h}$ at $37^{\circ} \mathrm{C}$ and the mix then diluted $\times 5$ with sterile $\mathrm{H}_{2} \mathrm{O}$. Oligonucleotide sequences for PCR adapters and primers are those used in the original protocol.

PCR preamplification. Five microlitres of the diluted ligation mix was used as the template for the preamplification. PCR was performed with $30 \mathrm{ng}$ Eco-A and $30 \mathrm{ng}$ of $\mathrm{Mse}-\mathrm{C}$ primers with 0.31 units Expand High Fidelity PCR System (Roche Applied Science) and $0.2 \mathrm{~mm}$ each dATP, dCTP, dGTP, dTTP with $1 \mathrm{mM} \mathrm{MgCl}_{2}$ in a total of $20 \mu \mathrm{L}$ of the manufacturer's buffer (composition confidential). The PCR programme consisted of 28 cycles of $\left(94{ }^{\circ} \mathrm{C}\right.$ for $30 \mathrm{~s}, 56^{\circ} \mathrm{C}$ for $60 \mathrm{~s}, 72^{\circ} \mathrm{C}$ for $\left.60 \mathrm{~s}\right)$. PCR products were diluted $\times 10$ with sterile $\mathrm{H}_{2} \mathrm{O}$.

Selective AFLP amplification. Five Eco-ACG/Mse-CNN primer combinations were used for the selective amplification (where $\mathrm{N}$ represents any nucleotide). Mse-CNN primer extensions were CAG, CAT, CTG, CTC and CTA. The Eco-ACG primer carried a VIC fluorochrome (Applied Biosystems - ABI, Foster City, CA, USA).

Selective amplifications were performed using $20 \mathrm{ng}$ Eco-ACG primer and $25 \mathrm{ng}$ Mse-CNN primer with $0.16 \mathrm{U}$ Expand High Fidelity PCR System and $0.1 \mathrm{mM}$ each dATP, dCTP, dGTP, dTTP with $1 \mathrm{mM} \mathrm{MgCl}_{2}$ in a total of $10 \mu \mathrm{L}$ of the manufacturer's buffer. The PCR programme consisted 36 cycles in total: 13 cycles of $\left(94{ }^{\circ} \mathrm{C}\right.$ for $30 \mathrm{~s}, t^{\circ} \mathrm{C}$ for $30 \mathrm{~s}, 72{ }^{\circ} \mathrm{C}$ for $60 \mathrm{~s}$, where $t$ drops from 65 to 56 in $0.7^{\circ} \mathrm{C}$ steps), followed by 23 cycles of $\left(94{ }^{\circ} \mathrm{C}\right.$ for $30 \mathrm{~s}, 56^{\circ} \mathrm{C}$ for $30 \mathrm{~s}, 72{ }^{\circ} \mathrm{C}$ for $\left.60 \mathrm{~s}\right)$.

For analysis of the selective AFLP-PCR products, $2 \mu \mathrm{L}$ of PCR product was mixed with $12 \mu \mathrm{L} \mathrm{Hi-Di}$ Formamide (ABI) $0.5 \mu \mathrm{L}$ Liz-500 size standard (ABI). Samples were denatured at $94^{\circ} \mathrm{C}$ for $3 \mathrm{~min}$ and electrophoresis performed using an ABI3130x1 genetic analyser according to the manufacturer's instructions. Fragment sizes were determined with reference to the size standard using GENEMAPPER v 4.0 (ABI). Fragment presence or absence was scored by hand and subsequently checked by a second 
investigator without reference to sample ID. A binary matrix of band presence/absence was then created. Each set of 96 reactions included two positive (known genotype duplicates) and two negative $\left(\mathrm{H}_{2} \mathrm{O}\right.$ or PCR mix without DNA) controls carried from restriction digest through to selective AFLP-PCR. Any band occurring in a sample was excluded from the analysis if it also occurred in a negative control. The error rate calculated between duplicates was 3.5\%. A total of 337 polymorphic AFLP markers were scored over 114 individuals. Only those markers with a maximum allele frequency of $95 \%$ or less were included in the final analyses.

\section{Data analysis}

For seedling establishment data, drought vs. control and warming vs. control comparisons were performed using paired-sample $t$ tests, with the total number of emerged seedlings per treatment as the response variable, and cohorts (years) as replicates. Transformation $[\log (x+1)]$ of the number of emerging seedlings was applied to attain normality.

Outlier loci were identified using the program DFDIST (M. A. Beaumont, 2005, programme distributed by the author), a dominant marker version of the program FDIST (Beaumont \& Nichols, 1996). DFDIST was used to perform pair-wise comparison of populations establishing in drought, warming and control treatment samples based on a two-stage procedure. 100000 realizations were performed based on a two deme, two population model, a maximum allowable allele frequency of 0.95 pooled across samples and the average $F_{\text {st }}$ calculated across all loci that met the 0.95 allele frequency criterion. We chose the 0.995 or 0.005 quantiles of the simulated distribution to define an envelope within which $99 \%$ of the data points are expected to lie. Any loci occurring outside these limits were designated as potential outliers. The average $F_{\mathrm{st}}$ was then recalculated without these outlier loci and a second round of simulations based on the adjusted average $F_{\text {st }}$ was used to identify outliers subject to natural selection. Given the allele frequency criterion of 0.95 , the number of polymorphic markers used in each outlier analysis was as follows: control vs. drought, 182; control vs. warming, 175; drought vs. warming, 191. Loci were assumed to be unlinked for the purpose of the analysis.

\section{Results}

Over the 7-year period 1999-2005, mean yearly $F$. thymifolia seedling density per treatment was significantly reduced in drought and warming treatments when compared with the control treatment $(t=2.93, P=0.026$, $\mathrm{df}=6 ; t=3.14, P=0.020, \mathrm{df}=6$, respectively, Fig. 2).

In genetic comparison of individuals establishing in drought and control treatments, five loci were identified that did not conform to the expectation of selective neutrality (Fig. 3a). These are candidate loci for marking regions of the $F$. thymifolia genome subject to natural selection (Vitalis et al., 2001; Beaumont, 2005; Storz, 2005). Genetic differentiation $\left(F_{\mathrm{st}}\right)$ at these loci ranged from 0.1361 to 0.2444 compared with mean $F_{\text {st }}$ across all neutral loci of 0.0066 (Fig. 3a). In the warming vs. control treatment comparison, two putatively nonneutral loci were detected, corresponding to two of the outliers detected in the drought vs. control comparison (Fig. $3 b$ ). $F_{\text {st }}$ at both of these outlying loci was 0.1787 , in comparison with a mean value of 0.0248 across all neutral loci. No outlying loci were detected in the drought vs. warming comparison, for which the mean $F_{\text {st }}$ across all loci was 0.0165 (Fig. 3c). We note, however, that low establishment has reduced the power to detect outlying loci in comparisons involving the warming treatment due to low sample number and, consequently, comparatively high genome wide $F_{\text {st }}$ (Murray \& Hare, 2006). Although criticised for being overly conservative in studies such as that presented here (Moran, 2003), if sequential Bonferroni corrections are applied to account for multiple tests (loci), three loci in the drought vs. control comparison remain as statistically significant outliers at $\alpha=0.05$ (loci 153L07, 158L10, L259L91, Fig. 3a). However, loci 153L07 and 158L10 display similar $F_{\mathrm{st}}$ and heterozygosity in all of the treatment comparisons (Fig. 3), suggesting that these loci may be located in the same region of the F. thymifolia genome.

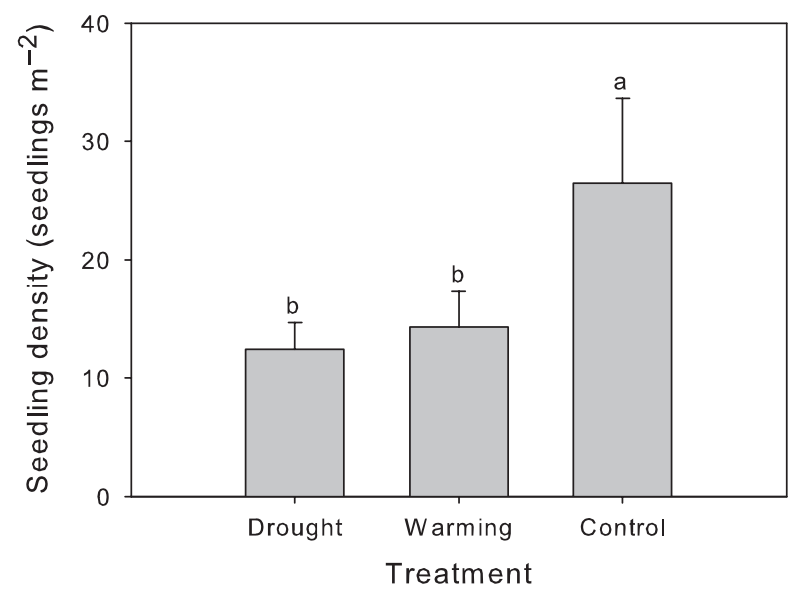

Fig. 2 Mean yearly Fumana thymifolia seedling density per treatment for the period 1999-2005. Error bars represent one standard error; treatments bearing the same letter do not differ significantly at $P<0.05$, based on paired-sample $t$ tests with cohorts (years) as replicates. 


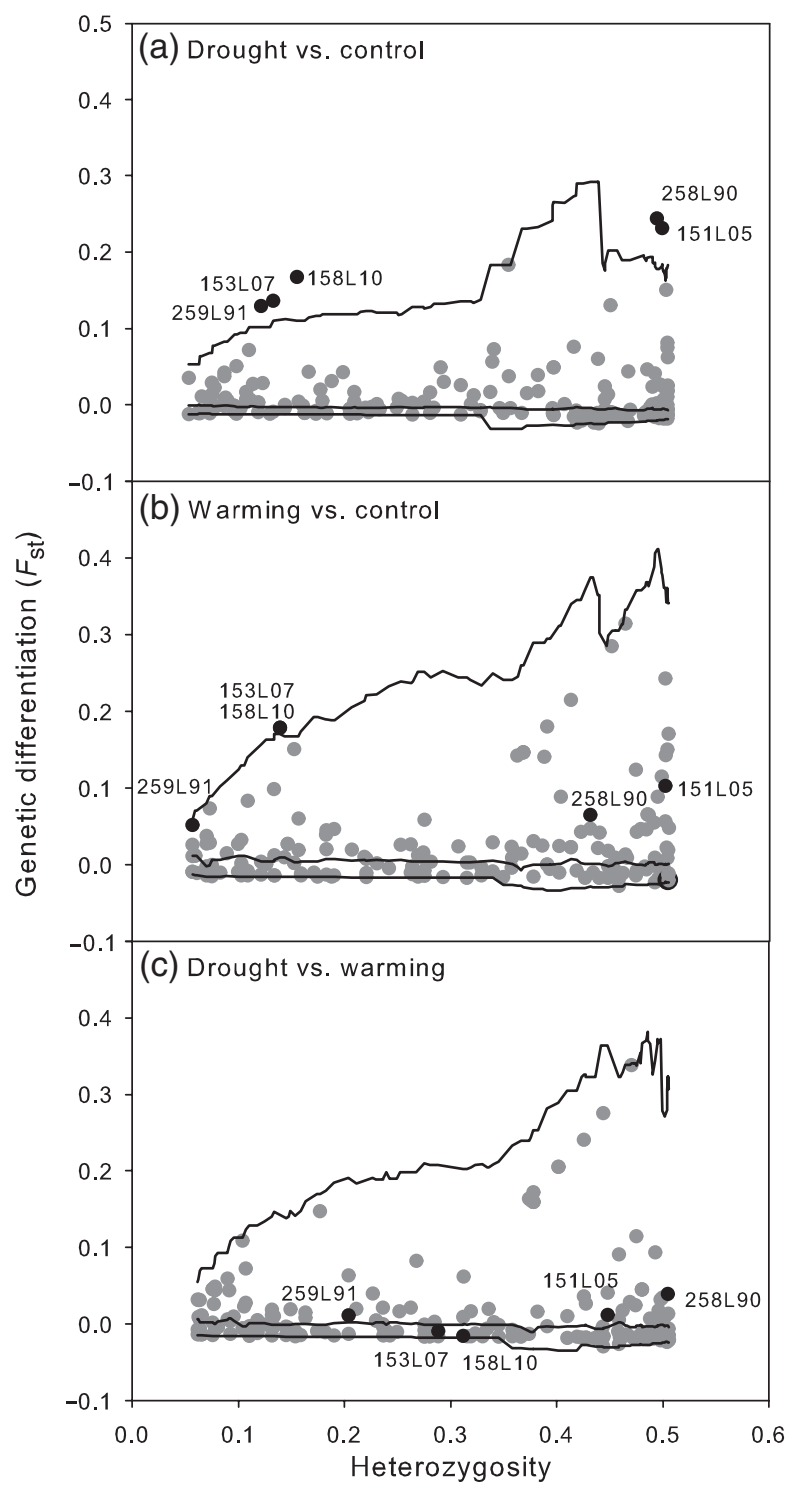

Fig. 3 Pair-wise comparison of the distribution of per-locus genetic differentiation $\left(F_{\mathrm{st}}\right)$ of Fumana thymifolia samples from drought, warming and control treatments. Values of $F_{\text {st }}$ derived from AFLP loci are plotted as a function of heterozygosity. Solid lines denote $0.995,0.5$ (median) and 0.005 quantiles of the conditional distribution obtained from coalescent simulations. The nonneutral loci detected in the drought vs. control comparison are labelled and presented in black; $P$ values for these loci are as follows: drought vs. control; 153L07, <0.00001; 158L10, $<0.00001$; 259L91，0.00025; 258L90, 0.001; 151L05, 0.00125; drought vs. warming; 153L07/158L10, 0.002999.

\section{Discussion}

Our results suggest that the significant reduction of F. thymifolia seedlings we observe in drought and warming treatments results from an episode of selection for individuals tolerant of the modified climatic conditions and is not due simply to a random reduction in plant establishment. Enhanced drought is implicated as the selection pressure responsible for the elevated differentiation of loci that occurs in individuals establishing in both the drought and warming treatments, since warming also increases water loss through enhanced evapotranspiration (Beier et al., 2004; Peñuelas et al., 2004). The possibility that common loci are under selection in both the drought and warming treatments (Fig. 3a and b) is supported by the generally low differentiation of the outliers detected in the drought vs. control comparison when the warming and drought treatments are compared (Fig. 3c). An alternative explanation for these outlying loci is the detection of preexisting but unidentified selection linked to spatial variation in the environmental conditions of the study site, which coincides with the location of the plots for each treatment. However, given that homogeneity of sample plots was maximized at the start of the experiment and that the plots for each treatment were physically intermixed within the community (Fig. 1), the occurrence and detection of preexisting selection based on small-scale differences in habitat is unlikely.

The detection of nonneutral loci in response to simulated climate change in this natural plant community reinforces results reported by other authors that show that genetic variability for climate-related traits exists within natural plant populations [Hamrick \& Holden, 1979; Cobb et al., 1994; Kelly et al., 2003; Mitton \& Duran, 2004; Franks et al., 2007, see Jump \& Peñuelas (2005) for a recent review]. Critically, we demonstrate through controlled manipulation of a natural plant community that selection by climate may act upon such variability to induce rapid genetic change within a population. This work, therefore, highlights one of the unseen effects of climate change - that it is driving changes in gene frequency within natural plant populations. Such genetic changes are occurring on the same time scale as current climatic changes, based on preexisting genetic variability within populations (Jump \& Peñuelas, 2005; Thomas, 2005; Jump et al., 2006a; Reusch \& Wood, 2007).

The existence of climate-related genetic variation within natural populations is perhaps not surprising; since interannual fluctuations in climate are a normal occurrence, different genotypes of a species may be favoured in different years (Thomas, 2005; Jump et al., 2006a). Interannual environmental fluctuations may, therefore, be one process by which functional genetic variation is maintained within natural populations. Under a scenario of rapid climatic change however, such interannual fluctuations are superimposed on a directional warming trend; the effect of which is to drive a directional genetic change as we report here. This evolutionary response is likely to reduce variability at the selected loci as the beneficial allele spreads 
throughout the population (Storz, 2005). A potential side effect of such a selective sweep, is that it may reduce the population's ability to respond genetically to environmental fluctuations in the future, leading to unpredictable effects on a species' presence and abundance at the level of the plant community (Jump \& Peñuelas, 2005).

In populations that are able to respond to selection pressures imposed by climatic changes, this ability may increase their persistence beyond that predicted under a species-based climate envelope approach (Jump \& Peñuelas, 2005). Work conducted on the European white birch (Betula pendula Roth) suggested that the natural population studied should be able to tolerate changes in mean annual temperature of approximately $1{ }^{\circ} \mathrm{C}$ based on changes in gene frequency within the population. This tolerance of increased temperature should decrease the population's susceptibility to invasion by species better adapted to higher temperatures, and thereby increase its persistence when subject to climatic changes (Kelly et al., 2003). Like the population of F. thymifolia we investigate here, this $B$. pendula population was located in a core area of the species' geographical distribution and is not, therefore, likely to be subject to the evolutionary constraints, such as lack of relevant genetic variation, that are associated with species' range margins (Thomas, 2005). However, even where an evolutionary response to climatic changes can occur, this may not be sufficient to ensure the survival of the population (Etterson \& Shaw, 2001; Davis et al., 2005; Bradshaw \& Holzapfel, 2006; Jump et al., 2006a). For example, in the European beech (Fagus sylvatica L.), genetic variability of individuals related to mean annual temperature was reported recently within a range-edge population, yet significant changes in the growth and distribution of this species are presently underway (Peñuelas \& Boada, 2003; Jump et al., 2006a, b).

Nonneutral loci identified following climatic changes induced by the climatic manipulation experiments we investigated pose an exciting challenge for further research in this field. Such nonneutral loci provide tantalising evidence that rapid evolution can and does occur in plant populations subject to environmental changes. However, genome scan methodologies such as that implemented here are not without controversy, as recent studies investigating the effectiveness of such techniques for outlier detection demonstrate [Teshima et al., 2006; Thornton \& Jensen, 2007, see Bonin et al. (2006) for a discussion of the potential problems of this approach]. The identification of such loci is, therefore, only the first step towards the characterisation of the functional phenotypic differences between individuals in populations where such adaptive genetic differentiation occurs.
Ultimately, the behaviour of such nonneutral loci must be confirmed by validating the observed locusspecific genetic differentiation through additional ecological and/or genomic investigations (Luikart et al., 2003). This in turn highlights a key challenge that very few genomic resources are available in nonmodel organisms such as F. thymifolia (in fact, a search of the Entrez-nucleotide database, found at http:/ / www.ncbi. nlm.nih.gov, returns only three nucleotide sequences for this genus). However, this lack of genomic resources should not discourage the conducting of population genomic investigations in nonmodel species. Rather, population genomic investigations should turn to ecological methods, such as controlled environment studies, to assess the adaptive significance of any nonneutral loci identified. Once outlier behaviour has been confirmed, this justifies further genomic investigation, which aims to understand adaptive differentiation at the nucleotide level by ultimately identifying the selected mutations or genes responsible for differences in fitness observed between contrasting environments.

\section{Acknowledgements}

We are grateful to J. Garcia, P. Arus, W. Howad and technical staff at the Departament de Genètica Vegetal at the Institut de Recerca i Tecnologia Agroalimentàries (IRTA), Cabrils, Barcelona for use of their genetic analyser and assistance with laboratory work. We thank Mark Bulling and Tom Webb for discussions on statistical analysis. This work was funded under grants from the European Union (Contract 506675, ALARM), the Catalan Government (grant SGR2005-00312), the Spanish Government (grants CGL2004-01402/BOS, CGL2006-01293/BOS and CGL2006-04025/BOS) and Fundación BBVA.

\section{References}

Alward RD, Detling JK, Milchunas DG (1999) Grassland vegetation changes and nocturnal global warming. Science, 283, 229231.

Beaumont MA (2005) Adaptation and speciation: what can $F_{s t}$ tell us? Trends in Ecology \& Evolution, 20, 435-440.

Beaumont MA, Nichols RA (1996) Evaluating loci for use in the genetic analysis of population structure. Proceedings of the Royal Society of London Series B-Biological Sciences, 263, 16191626.

Beier C, Emmett B, Gundersen P et al. (2004) Novel approaches to study climate change effects on terrestrial ecosystems in the field: drought and passive nighttime warming. Ecosystems, 7, 583-597.

Boag PT, Grant PR (1981) Intense natural selection in a population of Darwin's finches (Geospizinae) in the Galápagos. Science, 214, 82-85.

Bonin A, Taberlet P, Miaud C, Pompanon F (2006) Explorative genome scan to detect candidate loci for adaptation along a gradient of altitude in the common frog (Rana temporaria). Molecular Biology and Evolution, 23, 773-783. 
Bradshaw WE, Holzapfel CM (2001) Genetic shift in photoperiodic response correlated with global warming. Proceedings of the National Academy of Sciences, USA, 98, 14509-14511.

Bradshaw WE, Holzapfel CM (2006) Evolutionary response to rapid climate change. Science, 312, 1477-1478.

Campbell D, Bernatchez L (2004) Generic scan using AFLP markers as a means to assess the role of directional selection in the divergence of sympatric whitefish ecotypes. Molecular Biology and Evolution, 21, 945-956.

Castroviejo S, Aedo C, Cirujano S et al. (eds) (1993) Flora Iberica, Vol. III. Real Jardín Botánico, CSIC, Madrid.

Cobb N, Mitton JB, Whitham TG (1994) Genetic variation associated with chronic water and nutrient stress in pinyon pine. American Journal of Botany, 81, 936-940.

Davis MB, Shaw RG (2001) Range shifts and adaptive responses to quaternary climate change. Science, 292, 673-679.

Davis MB, Shaw RG, Etterson JR (2005) Evolutionary responses to changing climate. Ecology, 86, 1704-1714.

Etterson JR, Shaw RG (2001) Constraint to adaptive evolution in response to global warming. Science, 294, 151-154.

Franks SJ, Sim S, Weis AE (2007) Rapid evolution of flowering time by an annual plant in response to a climate fluctuation. Proceedings of the National Academy of Sciences of the United States of America, 104, 1278-1282.

Grant PR, Grant R (2002) Unpredictable evolution in a 30-year study of Darwin's finches. Science, 296, 707-711.

Hamrick JL, Holden LR (1979) Influence of microhabitat heterogeneity on gene frequency distribution and gametic phase disequilibrium in Avena barbata. Evolution, 33, 521-533.

Jump AS, Hunt JM, Martínez-Izquierdo JA, Peñuelas J (2006a) Natural selection and climate change: temperature-linked spatial and temporal trends in gene frequency in Fagus sylvatica. Molecular Ecology, 15, 3469-3480.

Jump AS, Hunt JM, Peñuelas J (2006b) Rapid climate changerelated growth decline at the southern range-edge of Fagus sylvatica. Global Change Biology, 12, 2163-2174.

Jump AS, Peñuelas J (2005) Running to stand still: adaptation and the response of plants to rapid climate change. Ecology Letters, 8, 1010-1020.

Kelly CK, Chase MW, de Bruijn A, Fay MF, Woodward FI (2003) Temperature-based population segregation in birch. Ecology Letters, 6, 87-89.

Lewis-Smith RI (1994) Vascular plants as bioindicators of regional warming in Antarctica. Oecologia, 99, 322-328.

Lloret F, Penuelas J, Estiarte M (2004) Experimental evidence of reduced diversity of seedlings due to climate modification in a Mediterranean-type community. Global Change Biology, 10, 248-258.

Luikart G, England PR, Tallmon D, Jordan S, Taberlet P (2003) The power and promise of population genomics: from genotyping to genome typing. Nature Reviews Genetics, 4, 981-994.

Mitton JB, Duran KL (2004) Genetic variation in pinon pine, Pinus edulis, associated with summer precipitation. Molecular Ecology, 13, 1259-1264.

Moran MD (2003) Arguments for rejecting the sequential Bonferroni in ecological studies. Oikos, 100, 403-405.

Murray MC, Hare MP (2006) A genomic scan for divergent selection in a secondary contact zone between Atlantic and
Gulf of Mexico oysters, Crassostrea virginica. Molecular Ecology, 15, 4229-4242.

Parmesan C, Ryrholm N, Stefanescu C et al. (1999) Poleward shifts in geographical ranges of butterfly species associated with regional warming. Nature, 399, 579-583.

Peñuelas J, Boada M (2003) A global change-induced biome shift in the Montseny mountains (NE Spain). Global Change Biology, 9, 131-140.

Peñuelas J, Gordon C, Llorens L et al. (2004) Non-intrusive field experiments show different plant responses to warming and drought among sites, seasons and species in a North-South European gradient. Ecosystems, 7, 598-612.

Réale D, McAdam AG, Boutin S, Berteaux D (2003) Genetic and plastic responses of a northern mammal to climate change. Proceedings of the Royal Society of London, Series B, 270, 591-596.

Reusch TBH, Wood TE (2007) Molecular ecology of global change. Molecular Ecology, 16, 3973-3992.

Savolainen V, Anstett MC, Lexer C et al. (2006) Sympatric speciation in palms on an oceanic island. Nature, 441, 210-213.

Storz JF (2005) Using genome scans of DNA polymorphism to infer adaptive population divergence. Molecular Ecology, 14, 671-688.

Strauss SY, Lau JA, Schoener TW, Tiffin P (in press) Evolution in ecological field experiments: implications for effect size. Ecology Letters, doi: 10.1111/j.1461-0248.2007.01128.x.

Teshima KM, Coop G, Przeworski M (2006) How reliable are empirical genomic scans for selective sweeps? Genome Research, 16, 702-712.

Thomas CD (2005) Recent evolutionary effects of climate change. In: Climate Change and Biodiversity (eds Lovejoy TE, Hannah L), pp. 75-88. Yale University Press, Cambridge, MA.

Thornton KR, Jensen JD (2007) Controlling the false-positive rate in multilocus genome scans for selection. Genetics, 175, 737-750.

Umina PA, Weeks AR, Kearney MR, McKechnie SW, Hoffmann AA (2005) A rapid shift in a classic clinal pattern in Drosophila reflecting climate change. Science, 308, 691-693.

Vigouroux Y, McMullen M, Hittinger CT et al. (2002) Identifying genes of agronomic importance in maize by screening microsatellites for evidence of selection during domestication. Proceedings of the National Academy of Sciences, USA, 99, 9650-9655.

Vitalis R, Dawson K, Boursot P (2001) Interpretation of variation across marker loci as evidence of selection. Genetics, 158, 1811-1823.

Vos P, Hogers R, Bleeker M et al. (1995) AFLP - a new technique for DNA fingerprinting. Nucleic Acids Research, 23, 4407-4414.

Walther GR, Burga CA, Edwards PJ (eds) (2001) Fingerprints of Climate Change. Adapted Behaviour and Shifting Species Ranges. Kluwer, New York.

Warren MS, Hill JK, Thomas JA et al. (2001) Rapid response of British butterflies to opposing forces of climate and habitat change. Nature, 414, 65-68.

Watkinson AR (1997) Plant population dynamics. In: Plant Ecology (ed. Crawley MJ), pp. 359-400. Blackwell Science, Oxford.

Wilding CS, Butlin RK, Grahame J (2001) Differential gene exchange between parapatric morphs of Littorina saxatilis detected using AFLP markers. Journal of Evolutionary Biology, 14, 611-619.

Wooton JC, Feng X, Ferdig MT et al. (2002) Genetic diversity and chloroquinine selective sweeps in Plasmodium falciparum. Nature Reviews Genetics, 418, 320-323. 